\title{
Neurosyphilis: a case report
}

\author{
Tugce Toptan, Betul Ozdilek, Gulay Kenangil, Mustafa Ulker, Fusun Mayda Domac \\ Department of Neurology, Erenkoy Training and Research Hospital for Neurologic and Psychiatric Disorders, Istanbul, Turkey
}

\begin{abstract}
Syphilis is a multisystem chronic infection caused by treponema pallidum. It can cause psychiatric disorders including depression, mania, psychosis, personality changes, delirium and dementia. With the introduction of penicillin into practice, the number of cases with syphilis decreased and its incidence increased with AIDS and HIV seropositivity. In this article, we present a case of neurosyphilis that manifested itself with neuropsychiatric symptoms.

Key words: Dementia; general paresis; neurosyphilis; psychiatric manifestations.
\end{abstract}

Syphilis is a multisystem chronic infection caused Sby treponema pallidum support $[1,2]$. Although there is a decrease in the number of cases of syphilis with the introduction of penicillin into use, it is still an important cause of the sexually transmitted diseases in developing countries because of the increase of incidence of AIDS and HIV seropositivity in the world $[3,4]$.

Syphilis progresses into four stages if untreated as primary, secondary, latent and tertiary stages. Primary syphilis is characterized by a typical painless syphilitic ulcer called chancre seen at the inoculation region after an incubation period lasting for 2-3 weeks. Secondary syphilis appears weeks or months later in nearly $25 \%$ of untreated patients and lymphadenopathy, gastrointestinal abnormalities and central nervous system alterations are seen. At the end of the latent period, tertiary syphilis develops in $25 \%$ of the untreated patients. Tertiary syphilis is seen 1-30 years after primary infection.
This inflammatory disease progresses slowly as neurosyphilis or gummatous syphilis $[5,6]$.

Neurosyphilis is classified as early and late syphilis. Cerebrospinal fluid (CSF), meninges and vascular structures are involved in the early stages of neurosyphilis, while in the late stage; cerebral tissue and spinal cord parenchyma are affected $[7,8]$. Therefore, neurosyphilis can manifest with many different symptoms.

In this article, we present a case of neurosyphilis with progressive cognitive changes and intractable behavioral and psychiatric problems whose primary and secondary phases were not detected.

\section{CASE REPORT}

A 40-year-old male patient was admitted to our hospital with amnesia, nervousness, personality changes, hostile attitudes, aggressive behaviors, hallucinations and illusions with a history of one year. 
His symptoms started 3 years ago with complaints of unwillingness to move and loss of appetite. He was consulted by a psychiatrist and put on antipsychotic drugs with no benefit. In the following year, urinary and fecal incontinence and speech disorders began. His medical and family history were not remarkable. On his physical examination, necrotic unhealed and deeply seated wounds were detected on his left knee and big toe of his right foot. On neurological examination, his cooperation was poor and he was disoriented to place and time. He had dysarthria and his comprehension ability was restricted to single commands. On cranial nerve examination, right and left pupil size were 2 and $3 \mathrm{~mm}$, respectively with bilateral decrease in light reflexes. The patient had bilateral bradykinesia. His Romberg test was negative. Blood analyses including complete blood count, hepatic, renal, thyroid function tests, fasting blood glucose, electrolytes, erythrocyte sedimentation rate, $\mathrm{C}$-reactive protein were within normal limits. Markers of vasculitis were negative. Among serum serological tests, Treponema Pallidum hemagglutination assay (TPHA) was positive at 1/2560 dilution. Venereal Diseases Research Laboratories (VDRL) and Rapid Plasma Reagin (RPR) tests were also positive. Cranial magnetic resonance (MR) images were evaluated as global cerebral and cerebellar atrophy and sequela of trauma in subcortical white matter of the right parietal lobe without contrast enhancement (Figure 1). Lumbar puncture was performed. Cerebrospinal fluid (CSF) analysis revealed the following results. Glucose was $67 \mathrm{mg} / \mathrm{dL}$, protein
$36 \mathrm{mg} / \mathrm{dL}$, Pandy test positivity and leukocyte 3/ $\mathrm{mm}^{3}$. In serologic examination of CSF, fluorescent treponemal antibody absorption (FTA- ABS) and VDRL was positive at $1 / 2$ and TPHA at $1 / 10240$ dilutions. The patient was diagnosed as neurosyphilis and after consultation with the department of infectious diseases, intravenous crystalized penicilline $\mathrm{G}$ (24 million units/day) was administered for 21 days. He was consulted with the department of psychiatry for the behavioral disorders and agitation. He was diagnosed as organic brain syndrome and offered risperidone $(3 \mathrm{mg} / \mathrm{d})$, haloperidol (10 $\mathrm{mg} / \mathrm{d})$ and carbamazepine $(800 \mathrm{mg} / \mathrm{d})$ therapy. Control CSF tests performed 6 months later were reported as TPHA positivity at 1/64 dilution, VDRL positivity and RPR $27.54 \mathrm{~s} / \mathrm{co}$. During his follow-up, we did not observe any improvement in his psychiatric symptoms, cognitive functions, urinary and fecal incontinence.

\section{DISCUSSION}

Neurosyphilis has been a rarely seen clinical entity within the last decade. It can be encountered in almost all psychiatric disorders including dementia, personality changes, mania, depression, psychosis and delirium.

In our patient, psychiatric manifestations started with introversion followed by gradually worsening cognitive decline, psychotic symptoms, dysarthria, urinary and fecal incontinence. His medical history did not reveal any clinical and dermatological complaints and signs peculiar to primary and secondary
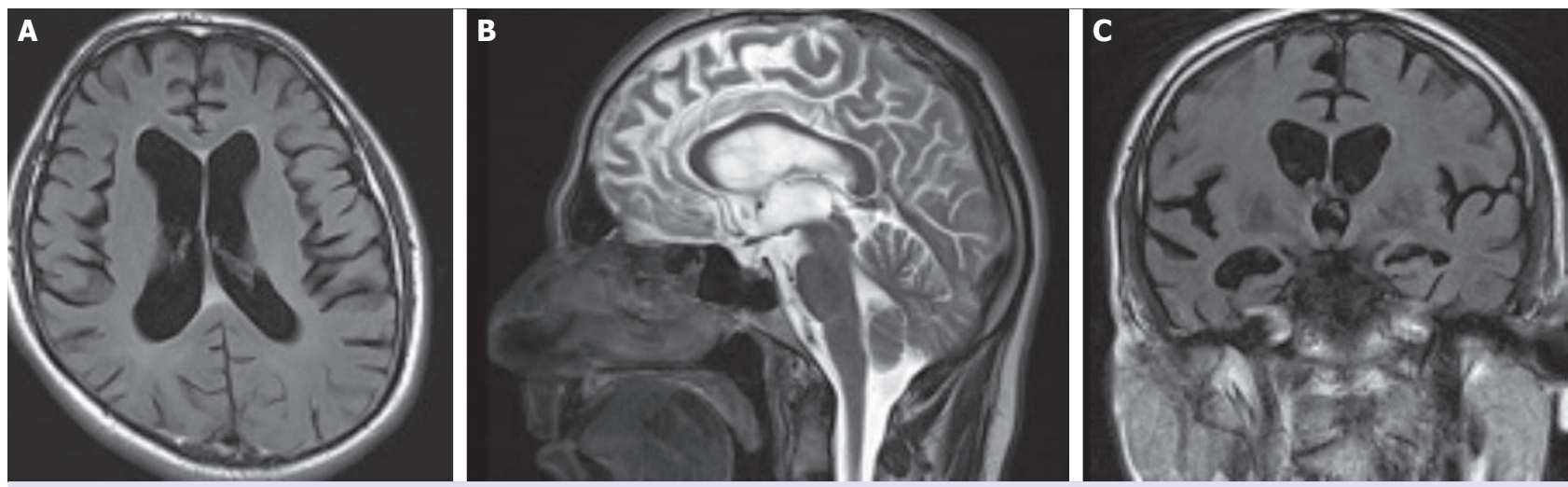

FIGURE 1. Flair-weighted axial section (A), T2-weighted sagittal section (B) and T1-weighted coronal section (C) of cranial MR images show diffuse cerebral, cerebellar atrophy and ventricular enlargement secondary to atrophy. 
stages of syphilis. Differential diagnosis of the patient involved treatable causes of cognitive dysfunction and psychiatric disorders, primary degenerative dementia and vascular disease. Creutzfeldt-Jakob disease was ruled out because of negative radiological and electroencephalographic findings. Primary dementia was discarded because of the patient age and development of cognitive decline within a short time (1-2 years) and severe course. MRI was negative for cerebrovascular disease, space-occupying mass lesion and vascular dementia. There was no sign for substance abuse in his medical history. Normal blood tests results, negative hepatitis and HIV serology ruled out metabolic and infectious causes of dementia. Serologic tests of serum and CSF for syphilis were found positive. Clinical findings and positive serologic results were compatible with general paresis, one of the forms of neurosyphilis.

Tertiary syphilis is a form of progressive dementia, also termed as general paresis, paretic neurosyphilis or dementia paralytica. Generally it develops 10-25 years after onset of the infection. In early phases of the disease, general paresis is associated with amnesia and personality changes. Most frequently problems related to memory and reasoning have been encountered. Less frequently, symptoms as depression, mania and psychosis are seen. Neurological examination may be normal or there are some pathological findings such as dysarthria, Argyl Robertson pupil defects, hypotonia, intentional tremor and reflex abnormalities [9]. Literature reports indicate that outcome of patients with general paresis is poor and death occurred within nearly 2.5 years before the era of penicillin treatment [7].

Effective treatment for neurosyphilis is high dose of intravenous crystallized penicillin. Treatment response should be examined with CSF analyses. After a treatment period, quantitative evaluation of blood serology is performed at 3-month-intervals and a decrease in the level of positivity in serologic tests can be observed. CSF should be analyzed at $6^{\text {th }}$ and $12^{\text {th }}$ months. Follow-up with neurological examination and CSF analysis can be stopped after the patient is cured, clinical stabilization is achieved and CSF serologic tests return to normal limits [10]. Treatment response is less frequently obtained in patients with general paresis when compared with cases with meningitis and meningovascular syphilis.
Indeed, irreversible neuronal damage is seen in general paresis, while the others reflect types of CNS inflammation [11].

As the case presented here is in the advanced stage of neurosyphilis, no improvement was observed in cognitive functions, psychotic manifestations and symptoms of dysarthria, urinary and fecal incontinence despite penicillin treatment. Nowadays, it should be remembered that syphilis in early stages can be overlooked, left untreated and can lead to irreversible manifestations, especially in developing countries.

Conflict of Interest: No conflict of interest was declared by the authors.

Financial Disclosure: The authors declared that this study has received no financial support.

\section{REFERENCES}

1. Bharucha NE. Infections of the nervous system. In: Bradley WG, Daroff RB, Fenichel GM (editors). Neurology in Clinical Practice. $3^{\text {th }}$ edition. London: Butterworth - Heinemann 2000;1334-5.

2. Adams RD, Victor M, Ropper AH (editors). Principles of Neurology. $7^{\text {th }}$ edition, New York: Mc Graw-Hill Companies 2000;722-8.

3. Schmidt RP. Neurosyphilis. In: Joynt RJ (editor). Clinical Neurology Vol.2 Revised edition. Philadelphia: Lippincott Williams \& Wilkins 1992;1-23.

4. Berger JR. Neurosyphilis in human immunodeficiency virus type 1-seropositive individuals. A prospective study. Arch Neurol 1991;48:700-2. CrossRef

5. Birnbaum NR, Goldschmidt RH, Buffett WO. Resolving the common clinical dilemmas of syphilis. Am Fam Physician 1999;59:2233-46.

6. Polsky I, Samuels SC. Neurosyphilis. Screening does sometimes reveal an infectious cause of dementia. Geriatrics 2001;56:60-2.

7. Merritt HH, Adams RD, Solomon HC. Neurosyphilis, Oxford, New York 1946.

8. Stokes JH, Beerman H, Ingraham NR. Modern Clinical Syphilology: Diagnosis, Treatment, Case Study, $3^{\text {rd }}$ ed, WB Saunders, Philadelphia 1944.

9. Marra CM. Neurosyphilis. http://www.uptodate.com/contents/neurosyphilis.

10. Stefanis L, Rowland LP. Infectious of the nervous system. In: Rowland LP, Pedley TA (editors). Merritt's Neurology. Lippincott Williams \& Wilkins, Philadelphia 2010;215-2.

11. Saddock BJ, Saddock VA. Klinik Psikiyatri. Aydın H, Bozkurt A (Çeviri ed.) 8. Baskı, Ankara: Güneş Kitabevi Ltd. Şti., 2007;454. 